\title{
Solving the hazmat transport network design problem
}

\author{
Erhan Erkut ${ }^{\mathrm{a}}$, Fatma Gzara ${ }^{\mathrm{b}, *}$ \\ ${ }^{\text {a }}$ Faculty of Business Administration, Bilkent University 06800, Ankara, Turkey \\ ${ }^{\mathrm{b}}$ School of Administrative Studies, York University, 4700 Keele Street, Toronto, Ont., Canada M3J 1P3
}

Available online 8 January 2007

\begin{abstract}
In this paper, we consider the problem of network design for hazardous material transportation where the government designates a network, and the carriers choose the routes on the network. We model the problem as a bilevel network flow formulation and analyze the bilevel design problem by comparing it to three other decision scenarios. The bilevel model is difficult to solve and may be ill-posed. We propose a heuristic solution method that always finds a stable solution. The heuristic exploits the network flow structure at both levels to overcome the difficulty and instability of the bilevel integer programming model. Testing on real data shows that the linearization of the bilevel model fails to find stable solutions and that the heuristic finds lower risk networks in less time. Further testing on random instances shows that the heuristically designed networks achieve significant risk reduction over single-level models. The risk is very close to the least risk possible. However, this reduction in risk comes with a significant increase in cost. We extend the bilevel model to account for the cost/risk trade-off by including cost in the first-level objective. The biobjective-bilevel model is a rich decision-support tool that allows for the generation of many good solutions to the design problem. (C) 2006 Elsevier Ltd. All rights reserved.
\end{abstract}

Keywords: Hazmat network design; Bilevel programming; Heuristic solution method

\section{Introduction}

Large quantities of hazardous materials (hazmats) are shipped on North American highways every day. While there is some level of risk involved with any movement of goods, hazmat shipments are associated with significant levels of risk, due to the nature of the cargo. For example, an accident involving a gasoline truck can result in a major fire on a highway and risk the lives of many drivers. Perhaps more serious is an accident involving a chlorine truck, which can result in injuries to individuals near the accident and the evacuation of the population nearby. Despite the good record of the industry, the public remains concerned about hazmat shipments, due to the low probability but high consequence of the risks. One way authorities mitigate hazmat transport risks is to confine them to a subset of available roads. Most cities designate "dangerous goods routes" for hazmat trucks. This paper deals with the design of a hazmat transport network both in and through a city.

In a city, hazmat trucks can be classified into two groups: (1) trucks that pass through, (2) trucks that originate from a supply location or are destined to a demand location in the city. While a simple ring road may be sufficient to route the hazmat trucks in group 1 away from the major population pockets, trucks in group 2 require a denser network that allows them access to major industrial areas. Once the hazmat network is designed, carriers are obliged to stay on the

\footnotetext{
* Corresponding author. Fax: +14167365963.

E-mail address: gzara@yorku.ca (F. Gzara).
} 
network as much as possible. Also, they need to take the shortest path between the network and the origin or destination point, if such points are not on the network.

In our design of a hazmat transport network, we consider the road network of a city, with a number of hazmat origins and destinations. There are two actors: the local government and the carriers. The local government will designate the network and the carriers will choose the routes. We can assume that the government is primarily interested in risk minimization, while the carriers are primarily interested in cost minimization. This situation gives rise to a bilevel design problem, with the government representing level 1 and the carriers representing level 2.

Once the government decides on the network, the carriers will take least-cost routes between the origin and destination on this network. Thus, one would expect the government to account for this behavior in designing the network. It makes no sense to design a network with the assumption that the carriers will stay on minimum risk routes. Also, the government usually cannot impose routes on carriers (not in a practical and economical way). Hence, the government should incorporate the carriers' rationale of choosing the least-cost routes into the design. This situation gives rise to the bilevel design problem in this paper.

Two main bodies of literature are relevant to this paper: bilevel transportation network design and hazmat transportation. Each area is rich with journal articles and book chapters. Bilevel transportation network design literature focus on traffic control and management where the outer level decision maker decides on such issues as design, control and improvement of the traffic network. The inner level decision makers plan their travel (route, mode of travel, origin and destination) based on the outer level decisions. One of the earlier work by Ben-Ayed et al. [1] focuses on highway network design. More recent applications consider traffic management [2], discrete road network expansion [3], and continuous network expansion choice [4]. For recent reviews we refer the reader to Yang and Bell $[5,6]$.

On the other hand, most of the literature on hazmat transportation focus on risk assessment, routing and facility location. For recent overviews on hazmat transportation, we refer the reader to Erkut et al. [7]. Hazmat network design has received the attention of researchers only recently. We discuss in detail the two papers that fall in this area. Erkut and Alp [8] consider a single-level network design problem. They restrict the network to a tree, so that there is a single path between each origin and destination. This restriction, in effect, removes the second level; the carriers have no alternate paths on the tree. They formulate the tree design problem as an integer programming problem with an objective of minimizing the total transport risk. Their model can be solved for moderate-sized problems using commercial solvers. They then develop a simple construction heuristic to expand the solution of the tree design problem by adding road segments. Such an addition relaxes the tree structure assumption and provides carriers with routing choices. Although costs are expected to decrease, risks may increase or decrease. The heuristic adds paths incrementally, which allows local authorities to trade off risk and cost.

Kara and Verter [9] is the first work to pose the hazmat network design problem as a bilevel problem. They formulate the design problem as a bilevel integer programming model, where hazmats are grouped into categories based on risk impact, and a network is designed for each group. It is assumed that the government imposes a different network for each category. Even though the bilevel model they propose includes all categories, there is no interaction between the shipments in different categories. So, the model naturally separates by category and a bilevel model can be solved for each category without affecting the overall solution. The researchers transform the bilevel program into a singlelevel mixed integer program by replacing the second-level problem by its KKT conditions and by linearizing the complementarity slackness constraints. In their computational experiments, they use Cplex to solve the model on an example with 48 vertices, 57 links, four categories and up to 22 origin-destination (OD) pairs. Although Cplex solves the single-level model efficiently, it fails to give a stable solution when an instance is ill-posed.

Although, we consider a similar problem to Kara and Verter [9], we generalize their model to the undirected case. That is, we allow the use of a road segment in both directions if it is used to one or more shipments in one direction. Most important, we protect the government from the worst case when the problem is ill-posed (i.e., when the linearized model fails to find a stable solution). We propose a heuristic solution method that exploits the network flow structure to overcome the difficulty of the bilevel integer programming model. The heuristic solves a set of network flow models iteratively and breaks cycles heuristically until a solution is obtained. We analyze the bilevel design problem by comparing it to three other decision scenarios. Testing on real data and on random instances shows that the heuristically designed networks achieve significant risk reduction over single-level models. The risk is very close to the least risk possible, implying that the heuristic finds network solutions that are very close to the bilevel optimal solution. A comparison of the heuristic algorithm to the solution of the linearized model by Cplex on real data proves the efficiency of the algorithm in terms of CPU time and quality of solution. 
The reduction in risk achieved using a bilevel model comes with a significant increase in cost. Whether or not this cost increase is acceptable depends on the decision makers. Perhaps the most comprehensive way to model the design problem is to include transport costs in the first-level objective. Although the main concern of the government may be public risk, it is reasonable to assume that they would be somewhat concerned about the financial cost imposed on the industry. We extend the bilevel model to account for the cost/risk trade-off by including cost in the first-level objective. The biobjective-bilevel model allows decision makers to trade off risk and cost. We believe this is the first paper to pose the biobjective-bilevel network design problem.

We discuss the alternative hazardous material network design scenarios in Section 2. We present the bilevel network design model in Section 3 and describe the solution procedure in Section 4. Section 5 gives our computational experience, where we also introduce the biobjective-bilevel model. We conclude the paper in Section 6.

\section{Four network design scenarios}

We analyze the network design problem assuming that the government aims to minimize risk and that the carriers aim to minimize cost. We consider four different scenarios which are differentiated by how the decisions of the two parties interact.

Scenario 1 (unregulated model): The government takes no action. The hazmat network is then determined by the carriers. This is the worst-case scenario from a risk perspective and may be unrealistic. Under this scenario, the hazmat network is determined by solving a multi-commodity network flow formulation with cost minimization as the objective. Denote the risk and cost associated with the minimum cost solution by $\mathrm{R}_{1}$ and $\mathrm{C}_{1}$, respectively.

Scenario 2 (over-regulated model): The government has the authority to impose certain routes on each carrier. This is the best-case scenario: the hazmat network and carrier routes are determined by the government. However, it implies over-regulation and may not be very realistic, as it requires monitoring every shipment. Nevertheless, it provides useful information, namely the lowest possible risk in the system. Under this scenario, the hazmat network is determined by solving a multi-commodity network flow formulation with risk minimization as the objective. Let $\mathrm{R}_{2}$ be the optimal risk level and $\mathrm{C}_{2}$ be its associated cost.

Scenario 3 (two-step model): The government has the authority to impose the same network on all carriers. Carriers then determine their minimum-cost routes. We assume that the government faces a single-level decision problem and does not account for the bilevel nature of the decision process. The hazmat network and risk level, in this case, are determined in two steps. First, the government finds a minimum risk network (which will be the same network as the one found under Scenario 2). Then, the carriers find minimum cost routes with a total cost of $\mathrm{C}_{3}$. The risk level $\mathrm{R}_{3}$ is thus the total risk associated with the flow of commodities on the minimum cost routes. One can think of this scenario as the more liberal (or less regulated) version of Scenario 2.

For the three scenarios, the minimum risk and minimum cost networks can be calculated exactly by solving a multicommodity network flow problem. We now provide a numerical example to illustrate that a multi-commodity network flow model is not a good representation of the design problem. Fig. 1 shows a symmetric graph of eight vertices and two commodities with OD pairs $(1,8)$ and $(2,8)$, respectively. For simplicity, assume that the cost for both commodities is the same. The risk and cost terms are shown in the figure. If the carrier is allowed to route freely (Scenario 1), it will select the minimum cost routes $\{(1,3),(3,8)\}$ and $\{(2,5),(5,6),(6,8)\}$ with a total cost of 6 units and total risk of 16 units. Under Scenario 2, the minimum risk routes are $\{(1,3),(3,6),(6,8)\}$ and $\{(2,5),(5,6),(6,7),(7,8)\}$ with a total risk of 11 units. Fig. $1 \mathrm{~b}$ shows the union of the two minimum risk paths. If the government designates this network as the hazmat transport network, but allows the carrier to choose its routes (Scenario 3), the latter will select the minimum cost routes $\{(1,3),(3,6),(6,8)\}$ and $\{(2,5),(5,6),(6,8)\}$ with a total cost of 8 units and total risk of 13 units. This risk is higher than what the government anticipates. Thus, by ignoring the effect of the carriers' choices on the actual risk level, the government finds a network that may result in far higher risk than is expected. In order to effectively manage risk, the government should include the response of the carriers in its decision problem. This leads to a fourth scenario.

Scenario 4 (bilevel model): The government considers the actual use of the hazmat network by the carriers. It faces a bilevel decision problem. Denote the risk by $\mathrm{R}_{4}^{*}$ and cost by $\mathrm{C}_{4}$.

It follows from the definition of the decision scenarios that $R_{2} \leqslant R_{4}^{*}, R_{4}^{*} \leqslant R_{3}$, and $R_{4}^{*} \leqslant R_{1}$. Thus, the over-regulated model risk $R_{2}$ is a lower bound on the bilevel optimal risk $R_{4}^{*} \cdot R_{3}$ and $R_{1}$ are valid upper bounds. The lower bound is valid when the bilevel model is solved heuristically, but the upper bounds are not. However, if a heuristic solution 

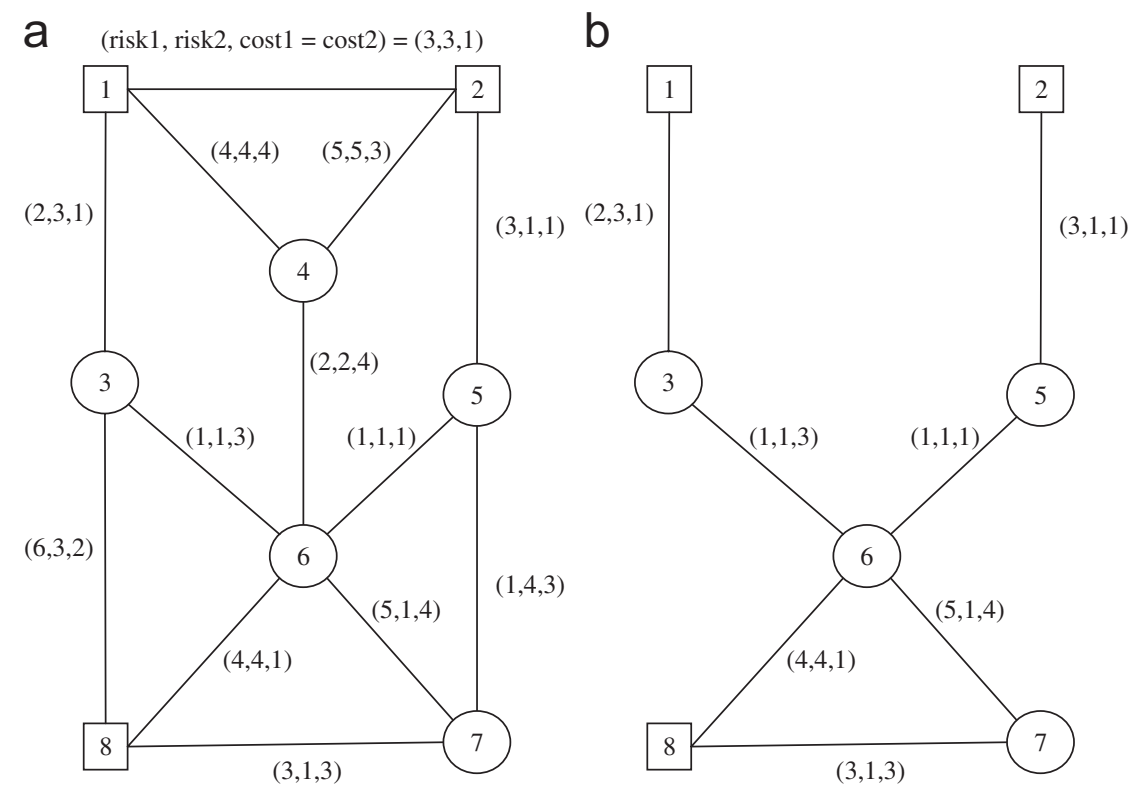

Fig. 1. An example demonstrating that a single-level model is insufficient.

leads to a risk higher than an available upper bound, the government is better off with the network that gives the lowest upper bound.

\section{The bilevel network design model}

The hazmat network design problem is a graph theoretical problem defined on a directed graph $G=(V, A)$, where $V$ is the set of vertices, and $A$ is the set of arcs of the graph. A vertex corresponds to a road intersection, and an arc corresponds to a road segment on the network. The network design problem finds a network to transport $K$ commodities between their respective origins and destinations. Each commodity corresponds to an OD pair. Let $(s(k), t(k))$ be the OD pair of commodity $k, k \in\{1 \ldots K\}$ and $d_{k}$ be the corresponding number of shipments. The parameters $r_{i j k}$ and $c_{i j k}$ refer to the risk and cost associated with a unit flow of commodity $k$ on arc $(i, j)$, respectively.

Let $x_{i j k}=1$, if arc $(i, j)$ is used by commodity $k$ in the optimal network. Binary variable $y_{i j}=1$ if arc $(i, j)$ or arc $(j, i)$ is in the network solution. The bilevel multi-commodity network flow model (BLF) is

$$
\begin{aligned}
& \text { BLF } \min _{y_{i j} \in\{0,1\}} \sum_{k \in\{1 \ldots K\}} \sum_{(i, j) \in A} d_{k} r_{i j k} x_{i j k} \\
& \text { s.t. } \quad y_{i j}=y_{j i} \\
& (i, j),(j, i) \in A \\
& x_{i j k} \in \arg \min \sum_{k \in\{1 \ldots K\}} \sum_{(i, j) \in A} d_{k} c_{i j k} x_{i j k} \\
& \sum_{i \in V} x_{i j k}-\sum_{i \in V} x_{j i k}=\left\{\begin{array}{ll}
-1 & \text { if } j=s(k), \\
1 & \text { if } j=t(k), \\
0 & \text { otherwise. }
\end{array} \quad j \in V, k \in\{1 \ldots K\}\right. \\
& x_{i j k} \leqslant y_{i j} \\
& (i, j) \in A, k \in\{1 \ldots K\} \\
& x_{i j k} \in\{0,1\} \\
& (i, j) \in A, k \in\{1 \ldots K\}
\end{aligned}
$$

The objective (1) of the government is to minimize the total risk on the network used by the carriers, while that of the carriers (3) is to minimize cost. Constraints (2) state that both $\operatorname{arcs}(i, j)$ and $(j, i)$ are open for use if any one of 
them is used by any of the shipments. Constraints (4) ensure the flow of commodity $k$ from its origin to destination. Constraints (5) ensure that only edges selected by the government can be used by the carriers. Constraints (6) are binary requirements on the variables.

BLF is similar to the bilevel model first proposed by Kara-Verter [9]. It is composed of two nested decision problems. The inner decision problem represents the decision of the carriers (follower) for some choice of the $(y)$ variables. The outer decision problem (ODP) is the decision of the government (leader). The constraint region of BLF is defined by the constraints of the inner and outer problems which is the set of feasible paths for all commodities on graph $G$. The feasible set of the carriers for each decision of the government is given by the set of feasible paths on a restricted graph defined by $A(y) \subseteq A$, the set of arcs of $A$ such that $y_{i j}=1$. The rational reaction set is the set of minimum cost paths of the carrier for each decision of the government. The set over which the government optimizes is the inducible region, the set of all feasible paths for the government and the carriers, such that minimum-cost paths are selected by the carriers, given the decision of the government. For comprehensive references on bilevel programming, we refer the reader to Bard [10] and Dempe [11].

On the one hand, BLF is well defined because any set of shortest paths between the OD pairs is an element of the constraint region. Moreover, the rational reaction set is nonempty for any network determined by the government, as the network itself is a feasible solution for the carriers. The carriers' decisions are not trivial, unless the network proposed by the government is acyclic.

On the other hand, BLF may be ill-posed, since the rational reaction set is not single-valued for all $y$. For example, refer to Fig. 1, and change the cost of edges (6-8) and (6-7) to 5 and 2, respectively. Then, the carriers have multiple minimum-cost paths. These solutions have different risk levels. For instance, if commodity 1 is assigned path $\{(1,3),(3,6),(6,7),(7,8)\}$ instead of $\{(1,3),(3,6),(6,8)\}$ the total risk increases by 4 units. Therefore, the government cannot induce the carriers to use the paths that achieve the lowest risk, and the problem does not have an optimal stable solution. In this case, it is preferable for the government to find a perturbed network with a small deviation from the best one and on which the risk of the carriers' network is stable.

It is worth noting that if the risks and costs are collinear (proportional): i.e. $r_{i j k}=\lambda \times c_{i j k}$, where $\lambda$ is a constant, the minimum-risk network also has minimum cost. Let $p_{1}$ and $p_{2}$ be two paths linking vertices $\hat{\imath}$ and $\hat{\jmath} \in V$. The risk associated with path $p_{l}, l \in\{1,2\}$, and with commodity $k$ is $R_{l k}=\sum_{(i, j) \in p_{l}} r_{i j k}$. Without loss of generality, let us assume that $R_{1 k} \leqslant R_{2 k}$. Then $C_{1 k} \leqslant C_{2 k}$, where $C_{l k}$ is the cost associated with path $p_{l}$ and commodity $k$, because $C_{1 k}=\lambda R_{1 k} \leqslant \lambda R_{2 k}=C_{2 k}$. In other words, in the presence of cycles, if there is a lower cost path, it also has a lower risk. So, the single-level decision of the government is optimal for the carriers, and the bilevel decision problem reduces to a single-level problem. Although these conditions trivialize the problem, it is highly unlikely that they will hold, given the problem context. It is safe to assume that travel costs will be linear functions of distance (for short distances, like those under consideration in this paper). Usually, accident frequencies are computed by multiplying the accident rate by the length of an edge. Hence, we can assume that the accident frequency is a linear function of distance. However, risk includes consequence and probability; the collinearity assumption will only hold if we are dealing with a constant consequence (as in constant population density along edges). Hence, we cannot, in general, assume that cost and risk will be collinear. The test instances used in this paper do not have collinear costs and risks and hence can be ill-posed.

\section{Heuristic solution of the bilevel model}

While BLF is a difficult problem, it is composed of single commodity shortest path problems that are easy to solve when isolated. We exploit this characteristic to propose a heuristic that solves the government and carrier decision problems iteratively on modified/restricted graphs.

The heuristic finds a network that minimizes the risk objective by solving a minimum risk path for each commodity. Call the resulting network $N$ with an associated risk value of $R$. If this network is passed to carriers, the actual risk value $A c t \_R$ corresponds to the paths chosen by the carriers. To calculate the actual risk, we solve a minimum cost path on $N$ for each commodity. If $R=A c t \_R$, then a solution is found. Otherwise, there is at least one commodity $k$ that does not use the same path as that proposed by the government. In order to eliminate the difference between the choices of the government and the carriers, we eliminate arcs iteratively. By eliminating arcs, we restrict the solution space of the government. The risk value $R$ is then expected to increase. This is not necessarily true for the cost value and the actual risk $A c t \_R$, because there is no (exclusion/inclusion) relationship between the consecutive networks, on which the carriers optimize. 
We propose two arc selection rules. The first rule finds an arc $(\hat{\imath}, \hat{\jmath})$ that is not used in the solution of the government but used in the solution of the carriers for some commodity $k$. In other words, commodity $k$ uses a different path than the one found under the minimum risk objective. Such an arc exists, because otherwise $A c t \_R=R$ and the algorithm stops. In case multiple arcs are available, the one with the highest risk is chosen (max-risk rule). The second selection rule uses the fact that the shortest path problem has the integrality property. This rule finds the candidate arcs in the same way as the first rule but selects an arc with the highest reduced risk (max-reduced-risk rule), where the reduced risk of an arc is the increase in total risk when the arc is included in the solution network. The steps of the algorithm follow.

Initialization: $t=0, A^{t}=A$, best_R $R=\infty$, set arc selection rule.

Iteration $t$ :

Step 1: Solve $K$ minimum-risk path problems on $A^{t}$.

$$
\begin{array}{ll}
\min & \sum_{(i, j) \in A\left(z^{t}\right)} d_{k} r_{i j k} x_{i j k} \\
\text { s.t. } & \sum_{i \in V} x_{i j k}-\sum_{i \in V} x_{j i k}= \begin{cases}-1 & \text { if } j=s(k), \\
1 & \text { if } j=t(k), \quad j \in V, \\
0 & \text { otherwise, } \\
& x_{i j k} \in\{0,1\}, \quad(i, j) \in A^{t} .\end{cases}
\end{array}
$$

Let $x_{k}^{t}$ and $R_{k}^{t}$ be the corresponding optimal solution and risk value for commodity $k$. Set $y_{i j}^{t}=1$ if $x_{i j k}^{t}=1$ or $x_{j i k}^{t}=1$. Let $A\left(y^{t}\right)$ be the network defined by $y^{t}$ with risk $R^{t}=\sum_{k \in\{1 \ldots K\}} R_{k}^{t}$.

Step 2: Solve $K$ minimum-cost path problems on $A\left(y^{t}\right)$

$$
\begin{array}{ll}
\min & \sum_{(i, j) \in A\left(z^{t}\right)} d_{k} c_{i j k} z_{i j k} \\
\text { s.t. } & \sum_{i \in V} z_{i j k}-\sum_{i \in V} z_{j i k}= \begin{cases}-1 & \text { if } j=s(k), \\
1 & \text { if } j=t(k), \quad j \in V, \\
0 & \text { otherwise, }\end{cases} \\
& z_{i j k} \in\{0,1\}, \quad(i, j) \in A\left(y^{t}\right) .
\end{array}
$$

Let $z^{t}$ and $C^{t}$ be the corresponding optimal solution and cost value and $A c t_{-} R^{t}$ be the risk associated with $z^{t}$. Update the best solution found: if $A c t \_R^{t}<B e s t_{-} R$ then Best_R $=A c t+R^{t}$

Step 3: If $A c t \_R^{t}=R^{t}$, then a potential heuristic network is determined by $z^{t}$ with risk level Act_R $R^{t}$. Go to Step 5.

Step 4: Let $(i, j)$ be an arc found according to the selection rule, remove $\operatorname{arcs}(i, j)$ and $(j, i)$ from the network, $t=t+1, A^{t+1}=A^{t}-\{(i, j),(j, i)\}$, and go to Step 1 .

Step 5: (Stability check) Define $\bar{A}$ the network determined by $y^{t}$ as

$$
\bar{A}=\left\{(i, j),(j, i): z_{i j k}^{t}=1 \text { or } z_{j i k}^{t}=1,(i, j) \in A\left(y^{t}\right), k \in\{1 \ldots K\}\right\}
$$

and solve NF_check on $\bar{A}$

$$
\begin{aligned}
& \text { NF_check } \max \sum_{k \in\{1 \ldots K\}} \sum_{(i, j) \in \bar{A}} d_{k} r_{i j k} x_{i j k} \\
& \text { s.t. } \sum_{i \in V} x_{i j k}-\sum_{i \in V} x_{j i k}=\left\{\begin{array}{ll}
-1 & \text { if } j=s(k), \\
1 & \text { if } j=t(k), \\
0 & \text { otherwise, }
\end{array} \quad j \in V, \quad k \in\{1 \ldots K\},\right. \\
& \sum_{k \in\{1 \ldots K\}} \sum_{(i, j) \in \bar{A}} d_{k} c_{i j k} x_{i j k} \leqslant C^{t}, \\
& x_{i j k} \in\{0,1\}, \quad(i, j) \in \bar{A}, k \in\{1 \ldots K\} .
\end{aligned}
$$

Let $\bar{R}$ be the objective value of NF_check. If $\bar{R}=A c t_{-} R^{t}$, then $\bar{A}$ is a stable heuristic network with risk Act_R $R^{t}$. Stop.

Else, $\bar{A}$ is not a stable network, and the actual risk may be as high as $\bar{R}$. Go to Step 4 . 
In Step 3, the condition $R^{t}=A c t_{-} R^{t}$ implies that the actual risk cannot be improved by removing more arcs. In the implementation, we use $\left(A c t_{-} R^{t}-R^{t}\right) / R^{t} \leqslant 1 e-5$ instead. When this condition holds, the network $\bar{A}$ associated with the minimum-cost solution $z^{t}$ and minimum cost $C^{t}$ is a heuristic solution that may be ill-posed. When $\bar{A}$ has cycles, there may be multiple minimum-cost solutions with different associated risk values. In that case, the risk is bigger than or equal to $A c t \_R^{t}$, because the latter is equal to the lowest possible risk $R^{t}$ on $A\left(z^{t}\right)$. To check whether or not $\bar{A}$ is stable, it is sufficient to check if the minimum-cost network flow problem defined on $\bar{A}$ has multiple solutions with different risk values. To verify this condition (Step 5), we solve the network flow problem (NF_check) with an upper bound constraint on the cost. The objective is to maximize risk. Let $\bar{R}$ be its optimal objective value. If $\bar{R}=A c t \_R^{t}$, then $\bar{A}$ is a stable heuristic network with risk $A c t \_R^{t}$. Else, $\bar{R}>R^{t}$ implies that there is at least one network on $\bar{A}$ with equal cost but higher risk. Hence $\bar{A}$ is not a stable solution, and the actual risk may be as much as $\bar{R}$, depending on the choice of the carriers. In this case and in order to protect the government from the worst case scenario, we continue the elimination of cycles until a stable solution is obtained. As a result of adding the upper bound constraint, NF_check does not separate into shortest path problems and does not have the integrality property anymore.

Note that although we consider the general case where the underlying road network is directed, the network determined by the government (and passed to the carriers) is undirected. For example, suppose that a street segment is two-way, but the inner problem finds only one of the directions. As the government cannot force the carriers to use a two-way street in only one direction, the network should include this edge in both directions. We account for this fact in the bilevel model and in the heuristic by constraints 2 . The model and heuristic handle one-way streets by setting the associated objective coefficients to a very large number.

\section{Numerical analysis}

We implement the heuristic algorithm in Matlab 6.5 and solve the network flow problems using Cplex 8.1. We perform all testing on a SunUltra Sparc 450 using data from the city of Ravenna, Italy [8]. The data provide information about the road network and about the origins and destinations of hazmats. The road network is composed of 105 vertices $(|V|=105)$ and 134 edges. We know the costs of traveling along the edges (actual distance), the population densities around the edges, and the locations and populations of places of assembly in the city (such as schools, churches, hospitals, factories, and office buildings). We use these data to calculate risk, which is measured as the product of the frequency of release in case of an accident and a population figure; the result is three risk measures: (1) on-arc, (2) around-arc, and (3) aggregate.

We calculate risk on-arc using population density along an edge and risk around-arc using population in its proximity. We calculate the aggregate risk measure, as in Erkut and Alp [8], by using the population in places of assembly within $500 \mathrm{~m}$ of the edges. The three risk measures are not generally collinear to the cost. Thus, the network design problem is not generally stable. The network flow models are directed. Hence, we create a directed network by associating two arcs with each edge. We perform testing on the OD pairs provided and on randomly generated pairs.

We perform testing in two phases and under the four decision scenarios discussed in Section 2. In phase 1, we investigate the significance of the bilevel model and we compare the heuristic to the solution of the linearized model by Cplex. In phase 2, we enrich the decision problem by adding a cost minimization objective to the first level. This enhancement recognizes that the government cannot be insensitive to the cost consequences of the network design. Carriers will have to pass increased costs on to consumers, and a more complete model should trade off costs and risks at the government level. In this phase, we extend BLF to a biobjective-bilevel formulation, where the outer objective minimizes a weighted sum of risk and cost.

In Section 5.1, for the first three scenarios discussed in Section 2, we calculate the minimum-risk and minimum-cost networks exactly by solving a multi-commodity network flow problem. For the fourth scenario, we find a heuristic solution using the heuristic described in Section 4. Let $\mathrm{R}_{4}$ and $\mathrm{C}_{4}$ be the heuristic solution's risk and cost, respectively.

In the tables, we provide the risk and cost measures by normalizing them via division by $\mathrm{R}_{4}$ and $\mathrm{C}_{4}$, the risk and cost of the bilevel design. For instance, $\mathrm{R}_{1} / \mathrm{R}_{4}$ is calculated as

$$
\mathrm{R}_{1} / \mathrm{R}_{4}=\frac{\mathrm{R}_{1}}{\mathrm{R}_{4}} \times 100 .
$$

In addition, we provide the number of iterations performed by the heuristic (which is equal to the number of edges removed), and the CPU time in seconds. 
Table 1

35-commodity instance results under different settings

\begin{tabular}{|c|c|c|c|c|c|c|c|c|c|c|c|}
\hline Risk & $\mathrm{R}_{1} / \mathrm{R}_{4}$ & $\mathrm{R}_{2} / \mathrm{R}_{4}$ & $\mathrm{R}_{3} / \mathrm{R}_{4}$ & $\mathrm{R}_{5} / \mathrm{R}_{4}$ & $\mathrm{C}_{1} / \mathrm{C}_{4}$ & $\mathrm{C}_{2} / \mathrm{C}_{4}$ & $\mathrm{C}_{3} / \mathrm{C}_{4}$ & $\mathrm{C}_{5} / \mathrm{C}_{4}$ & Iter & CPU-heur & CPU-Cplex \\
\hline \multicolumn{12}{|c|}{ Max-risk arc selection rule } \\
\hline Aggregate & 1742.96 & 98.97 & 103.92 & 103.92 & 50.03 & 114.87 & 87.09 & 87.09 & 3 & 6.88 & 28.60 \\
\hline Around-arc & 348.13 & 97.50 & 100.42 & 100.42 & 75.51 & 93.60 & 93.08 & 93.08 & 4 & 8.33 & 28.99 \\
\hline On-arc & 111.96 & 98.67 & 108.51 & 108.51 & 68.22 & 95.15 & 90.93 & 90.93 & 5 & 10.71 & 28.78 \\
\hline \multicolumn{12}{|c|}{ Max-reduced-risk arc selection rule } \\
\hline Aggregate & 1729.38 & 98.20 & 103.11 & 103.11 & 56.62 & 130.00 & 98.56 & 98.56 & 2 & 5.11 & 28.60 \\
\hline Around-arc & 349.76 & 97.96 & 100.89 & 100.89 & 81.03 & 100.45 & 99.89 & 99.89 & 5 & 10.19 & 28.99 \\
\hline On-arc & 113.47 & 100.00 & 109.98 & 109.98 & 71.69 & 99.99 & 95.55 & 95.55 & 2 & 5.49 & 28.78 \\
\hline
\end{tabular}
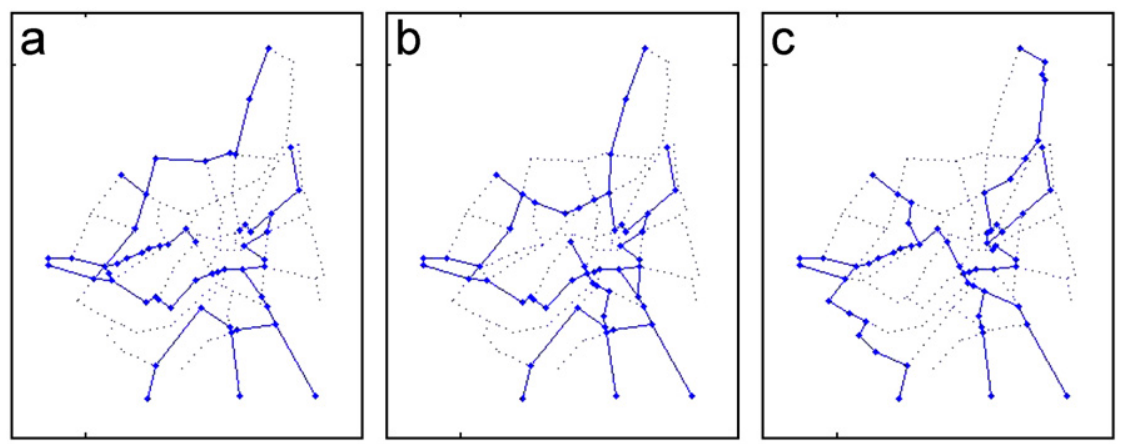

Fig. 2. Network solution of the 35-pairs example. (a) Aggregate risk measure, (b) around-arc risk measure, (c) on-arc risk measure.

Table 2

Origin-destination generation

\begin{tabular}{lcc}
\hline$K$ & Number of origin vertices & Origin repetition \\
\hline 20 & 5 & 56432 \\
30 & 6 & 756543 \\
40 & 8 & 97864321 \\
50 & 10 & 9877553321 \\
60 & 12 & 987665544321 \\
\hline
\end{tabular}

\subsection{Phase 1 Ravenna data}

In the Ravenna data, there are shipments between 35 different OD pairs, and the number of shipments between each OD pair is given. We solve the design problem under the four decision scenarios described earlier. We experiment with the three risk measures and the two arc selection rules. We set up the linearization of BLF by replacing the inner problem by its KKT conditions and by linearizing the complementarity slackness conditions. We then solve the linearized model using Cplex. Denote by $\mathrm{R}_{5}$ the actual risk associated with the Cplex solution and $\mathrm{C}_{5}$ its cost. Table 1 shows the results, and Fig. 2 gives selected solution networks. Figs. $2 \mathrm{a}-\mathrm{c}$ show the networks found by the heuristic under each of the risk measures with the max-reduced-risk rule.

Under all three risk measures, the linearized model solved by Cplex fails to produce a stable solution. All Cplex solutions correspond to minimum risk solutions (Scenario 1). When checked for stability, the actual risk and cost values are similar to those produced by Scenario 3. In summary, the heuristic finds stable network designs with lower levels of risk and in less CPU time then Cplex. The heuristic performs a small number of iterations and lasts a few seconds in all experiments. The number of iterations and CPU time used by the heuristic are comparable under both rules. $R_{1} / R_{4}$ 
Table 3

Detailed results on the random test problems using (a) the aggregate risk measure and the max-risk arc selection rule; (b) the aggregate risk measure and the max-reduced-risk arc selection rule

\begin{tabular}{|c|c|c|c|c|c|c|c|c|c|}
\hline$K$ & Run & $\mathrm{R}_{1} / \mathrm{R}_{4}$ & $\mathrm{R}_{2} / \mathrm{R}_{4}$ & $\mathrm{R}_{3} / \mathrm{R}_{4}$ & $\mathrm{C}_{1} / \mathrm{C}_{4}$ & $\mathrm{C}_{2} / \mathrm{C}_{4}$ & $\mathrm{C}_{3} / \mathrm{C}_{4}$ & Iter & CPU-time \\
\hline \multicolumn{10}{|l|}{ (a) } \\
\hline \multirow[t]{10}{*}{20} & 1 & 499.19 & 100 & 100 & 50.82 & 100 & 100 & 1 & 2.35 \\
\hline & 2 & 299.46 & 97.79 & 188.62 & 35.88 & 97.18 & 47.17 & 3 & 4.2 \\
\hline & 3 & 302.07 & 100 & 100 & 37.96 & 100.81 & 100 & 1 & 2.38 \\
\hline & 4 & 657.7 & 99.37 & 101.15 & 47.51 & 95.9 & 84.83 & 2 & 2.95 \\
\hline & 5 & 396.98 & 100 & 100 & 71.24 & 100.75 & 100 & 1 & 1.99 \\
\hline & 6 & 582.98 & 98.89 & 116.39 & 51.15 & 95.12 & 78.64 & 2 & 3.09 \\
\hline & 7 & 596.34 & 100 & 100 & 52.55 & 105.02 & 100 & 1 & 2.33 \\
\hline & 8 & 1255.83 & 100 & 100 & 46.83 & 103.13 & 100 & 1 & 2.44 \\
\hline & 9 & 467.98 & 99.28 & 103.93 & 54.1 & 95.53 & 81.43 & 3 & 4.04 \\
\hline & 10 & 302.32 & 100 & 100 & 65.07 & 100.74 & 100 & 1 & 2.54 \\
\hline \multirow[t]{10}{*}{30} & 1 & 478.88 & 98.57 & 119.52 & 55.79 & 99.96 & 85.58 & 2 & 4.74 \\
\hline & 2 & 703.98 & 100 & 100 & 49.96 & 102.28 & 100 & 1 & 3.7 \\
\hline & 3 & 270.56 & 100 & 100 & 46.34 & 109.88 & 100 & 1 & 3.91 \\
\hline & 4 & 412.39 & 98.4 & 100 & 61.15 & 118.1 & 100 & 3 & 6.3 \\
\hline & 5 & 818.49 & 98.9 & 189.85 & 44.3 & 99.84 & 73.25 & 2 & 4.72 \\
\hline & 6 & 397.91 & 100 & 100 & 45.56 & 101.98 & 100 & 1 & 4.24 \\
\hline & 7 & 691.52 & 100 & 100 & 45.72 & 101.85 & 100 & 1 & 3.63 \\
\hline & 8 & 312.83 & 100 & 100 & 54.82 & 104.9 & 100 & 1 & 3.53 \\
\hline & 9 & 287.23 & 100 & 100 & 46.41 & 102.74 & 100 & 1 & 3.62 \\
\hline & 10 & 608.94 & 100 & 100 & 48.04 & 104.68 & 100 & 1 & 3.82 \\
\hline \multirow[t]{10}{*}{40} & 1 & 552.91 & 99.44 & 103.81 & 51.52 & 105.09 & 88.35 & 3 & 9.37 \\
\hline & 2 & 425.74 & 97.78 & 122.47 & 52.44 & 102.69 & 82.16 & 4 & 11.03 \\
\hline & 3 & 395.97 & 99.59 & 128.76 & 43.29 & 102.13 & 83.99 & 2 & 7.06 \\
\hline & 4 & 701.07 & 89.33 & 244.94 & 44.46 & 101.3 & 77.07 & 3 & 8.57 \\
\hline & 5 & 657.84 & 100 & 100 & 48.97 & 104.37 & 100 & 1 & 4.94 \\
\hline & 6 & 423.47 & 99.31 & 133.2 & 47.99 & 98.46 & 79.87 & 2 & 6.81 \\
\hline & 7 & 839.12 & 100 & 100 & 47 & 106.81 & 100 & 1 & 5.13 \\
\hline & 8 & 756.98 & 97.13 & 130.92 & 51.62 & 92.52 & 74.65 & 2 & 6.25 \\
\hline & 9 & 450.79 & 97.19 & 120.41 & 36.4 & 94.01 & 79.63 & 2 & 6.88 \\
\hline & 10 & 626.04 & 100 & 100 & 51.04 & 103.46 & 100 & 1 & 5.33 \\
\hline \multirow[t]{10}{*}{50} & 1 & 870.57 & 100 & 100 & 50.99 & 101.79 & 100 & 1 & 6.63 \\
\hline & 2 & 311.73 & 100 & 100 & 47.52 & 102.04 & 100 & 1 & 6.25 \\
\hline & 3 & 533.6 & 98.96 & 134.41 & 46.9 & 99.94 & 82.29 & 2 & 9.36 \\
\hline & 4 & 538.49 & 92.4 & 265.63 & 47 & 95.07 & 63.69 & 3 & 11.45 \\
\hline & 5 & 576.22 & 99.04 & 135.92 & 43.9 & 103.15 & 82.78 & 2 & 9.41 \\
\hline & 6 & 1404.37 & 99.13 & 101.22 & 48.64 & 101.69 & 80.9 & 3 & 10.47 \\
\hline & 7 & 609.03 & 99.2 & 131.72 & 41.05 & 100.3 & 81.2 & 2 & 8.56 \\
\hline & 8 & 477.02 & 97.94 & 121.65 & 49.51 & 97.43 & 81.9 & 4 & 12.99 \\
\hline & 9 & 475.29 & 100 & 100 & 55.82 & 101.13 & 100 & 1 & 6.58 \\
\hline & 10 & 732.82 & 98.73 & 101.88 & 52.85 & 110.29 & 95.19 & 4 & 13.57 \\
\hline \multirow[t]{10}{*}{60} & 1 & 447.31 & 97.95 & 113.95 & 46.28 & 100.83 & 80.09 & 4 & 16.61 \\
\hline & 2 & 522.07 & 100 & 100 & 54.67 & 101.57 & 100 & 1 & 8.17 \\
\hline & 3 & 387.73 & 98.94 & 119.82 & 42.45 & 94.6 & 77.62 & 2 & 10.8 \\
\hline & 4 & 444.14 & 97.48 & 113.35 & 49.64 & 98.84 & 80.11 & 4 & 15.93 \\
\hline & 5 & 280.81 & 93.57 & 116.72 & 51.47 & 98.75 & 75.83 & 5 & 19.09 \\
\hline & 6 & 783.2 & 100 & 100 & 52.68 & 104.19 & 100 & 1 & 8.9 \\
\hline & 7 & 434.59 & 99.59 & 103.16 & 56.77 & 101.48 & 96.7 & 3 & 14.08 \\
\hline & 8 & 641.27 & 99.54 & 154.85 & 49.18 & 104.88 & 81.5 & 2 & 11.32 \\
\hline & 9 & 311.59 & 92.99 & 209.43 & 56.03 & 97.89 & 76.29 & 5 & 18.19 \\
\hline & 10 & 723.23 & 98.7 & 125.16 & 57.01 & 98.67 & 84.62 & 3 & 13.28 \\
\hline
\end{tabular}


Table 3 (Contd).

\begin{tabular}{|c|c|c|c|c|c|c|c|c|c|}
\hline K & Run & $\mathrm{R}_{1} / \mathrm{R}_{4}$ & $\mathrm{R}_{2} / \mathrm{R}_{4}$ & $\mathrm{R}_{3} / \mathrm{R}_{4}$ & $\mathrm{C}_{1} / \mathrm{C}_{4}$ & $\mathrm{C}_{2} / \mathrm{C}_{4}$ & $\mathrm{C}_{3} / \mathrm{C}_{4}$ & Iter & CPU-time \\
\hline \multicolumn{10}{|c|}{ (b) } \\
\hline \multirow[t]{10}{*}{20} & 1 & 499.19 & 100 & 100 & 50.82 & 100 & 100 & 1 & 2.12 \\
\hline & 2 & 158.77 & 51.85 & 100 & 76.06 & 206.01 & 100 & 3 & 4.24 \\
\hline & 3 & 302.07 & 100 & 100 & 37.96 & 100.81 & 100 & 1 & 2.39 \\
\hline & 4 & 657.7 & 99.37 & 101.15 & 47.93 & 96.74 & 85.57 & 2 & 2.96 \\
\hline & 5 & 396.98 & 100 & 100 & 71.24 & 100.75 & 100 & 1 & 2.06 \\
\hline & 6 & 582.98 & 98.89 & 116.39 & 51.15 & 95.12 & 78.64 & 2 & 3.18 \\
\hline & 7 & 596.34 & 100 & 100 & 52.55 & 105.02 & 100 & 1 & 2.26 \\
\hline & 8 & 1255.83 & 100 & 100 & 46.83 & 103.13 & 100 & 1 & 2.40 \\
\hline & 9 & 469.5 & 99.6 & 104.27 & 52.65 & 92.97 & 79.24 & 2 & 3.16 \\
\hline & 10 & 302.32 & 100 & 100 & 65.07 & 100.74 & 100 & 1 & 2.49 \\
\hline \multirow[t]{10}{*}{30} & 1 & 478.58 & 98.51 & 119.44 & 58.08 & 104.06 & 89.09 & 2 & 4.89 \\
\hline & 2 & 703.98 & 100 & 100 & 49.96 & 102.28 & 100 & 1 & 3.70 \\
\hline & 3 & 270.56 & 100 & 100 & 46.34 & 109.88 & 100 & 1 & 3.89 \\
\hline & 4 & 412.39 & 98.4 & 100 & 61.15 & 118.1 & 100 & 3 & 6.12 \\
\hline & 5 & 818.49 & 98.9 & 189.85 & 44.3 & 99.84 & 73.25 & 2 & 4.72 \\
\hline & 6 & 397.91 & 100 & 100 & 45.56 & 101.98 & 100 & 1 & 4.12 \\
\hline & 7 & 691.52 & 100 & 100 & 45.72 & 101.85 & 100 & 1 & 3.54 \\
\hline & 8 & 312.83 & 100 & 100 & 54.82 & 104.9 & 100 & 1 & 3.49 \\
\hline & 9 & 287.23 & 100 & 100 & 46.41 & 102.74 & 100 & 1 & 3.59 \\
\hline & 10 & 608.94 & 100 & 100 & 48.04 & 104.68 & 100 & 1 & 3.91 \\
\hline \multirow[t]{10}{*}{40} & 1 & 552.91 & 99.44 & 103.81 & 51.52 & 105.09 & 88.35 & 3 & 9.39 \\
\hline & 2 & 426.82 & 98.03 & 122.78 & 50.03 & 97.96 & 78.37 & 3 & 8.88 \\
\hline & 3 & 395.97 & 99.59 & 128.76 & 43.29 & 102.13 & 83.99 & 2 & 7.00 \\
\hline & 4 & 665.32 & 84.77 & 232.45 & 46.88 & 106.81 & 81.26 & 3 & 8.35 \\
\hline & 5 & 657.84 & 100 & 100 & 48.97 & 104.37 & 100 & 1 & 4.87 \\
\hline & 6 & 420.79 & 98.68 & 132.35 & 47.63 & 97.72 & 79.27 & 2 & 6.52 \\
\hline & 7 & 839.12 & 100 & 100 & 47 & 106.81 & 100 & 1 & 5.33 \\
\hline & 8 & 766.69 & 98.37 & 132.6 & 57.43 & 102.94 & 83.05 & 2 & 6.31 \\
\hline & 9 & 450.79 & 97.19 & 120.41 & 36.4 & 94.01 & 79.63 & 2 & 6.99 \\
\hline & 10 & 626.04 & 100 & 100 & 51.04 & 103.46 & 100 & 1 & 5.62 \\
\hline \multirow[t]{10}{*}{50} & 1 & 870.57 & 100 & 100 & 50.99 & 101.79 & 100 & 1 & 6.73 \\
\hline & 2 & 311.73 & 100 & 100 & 47.52 & 102.04 & 100 & 1 & 6.54 \\
\hline & 3 & 538.29 & 99.83 & 135.59 & 48.43 & 103.2 & 84.97 & 2 & 9.56 \\
\hline & 4 & 538.49 & 92.4 & 265.63 & 47 & 95.07 & 63.69 & 3 & 11.58 \\
\hline & 5 & 575.07 & 98.84 & 135.65 & 43.85 & 103.05 & 82.7 & 2 & 8.95 \\
\hline & 6 & 1392.89 & 98.32 & 100.39 & 52.55 & 109.86 & 87.4 & 4 & 13.12 \\
\hline & 7 & 599.26 & 97.61 & 129.61 & 39.76 & 97.13 & 78.64 & 2 & 8.65 \\
\hline & 8 & 478.36 & 98.21 & 121.99 & 54.6 & 107.46 & 90.33 & 4 & 13.26 \\
\hline & 9 & 475.29 & 100 & 100 & 55.82 & 101.13 & 100 & 1 & 6.53 \\
\hline & 10 & 733.26 & 98.79 & 101.94 & 50.14 & 104.64 & 90.31 & 3 & 11.11 \\
\hline \multirow[t]{10}{*}{60} & 1 & 453.97 & 99.41 & 115.65 & 48.9 & 106.53 & 84.61 & 4 & 16.28 \\
\hline & 2 & 522.07 & 100 & 100 & 54.67 & 101.57 & 100 & 1 & 8.29 \\
\hline & 3 & 387.73 & 98.94 & 119.82 & 42.45 & 94.6 & 77.62 & 2 & 11.00 \\
\hline & 4 & 448.94 & 98.53 & 114.58 & 51.34 & 102.22 & 82.85 & 3 & 13.70 \\
\hline & 5 & 282.19 & 94.03 & 117.29 & 49.74 & 95.44 & 73.28 & 4 & 16.61 \\
\hline & 6 & 783.2 & 100 & 100 & 52.68 & 104.19 & 100 & 1 & 9.00 \\
\hline & 7 & 432.01 & 99.01 & 102.55 & 55.49 & 99.19 & 94.53 & 4 & 16.66 \\
\hline & 8 & 641.27 & 99.54 & 154.85 & 49.18 & 104.88 & 81.5 & 2 & 11.21 \\
\hline & 9 & 311.89 & 93.08 & 209.63 & 56.73 & 99.1 & 77.24 & 5 & 18.27 \\
\hline & 10 & 729.03 & 99.49 & 126.17 & 58.35 & 100.98 & 86.61 & 4 & 15.57 \\
\hline
\end{tabular}


Table 4

Summary results on the random test problems using the aggregate risk measure

\begin{tabular}{|c|c|c|c|c|c|c|c|c|}
\hline$K$ & $\mathrm{R}_{1} / \mathrm{R}_{4}$ & $\mathrm{R}_{2} / \mathrm{R}_{4}$ & $\mathrm{R}_{3} / \mathrm{R}_{4}$ & $\mathrm{C}_{1} / \mathrm{C}_{4}$ & $\mathrm{C}_{2} / \mathrm{C}_{4}$ & $\mathrm{C}_{3} / \mathrm{C}_{4}$ & Iter & CPU-time \\
\hline \multicolumn{9}{|c|}{ Max-risk arc selection rule } \\
\hline 20 & 536.09 & 99.53 & 111.01 & 51.31 & 99.42 & 89.21 & 1.6 & 2.83 \\
\hline 30 & 498.27 & 99.59 & 110.94 & 49.81 & 104.62 & 95.88 & 1.4 & 4.22 \\
\hline 40 & 582.99 & 97.98 & 128.45 & 47.47 & 101.08 & 86.57 & 2.1 & 7.14 \\
\hline 50 & 652.91 & 98.54 & 129.24 & 48.42 & 101.28 & 86.79 & 2.3 & 8.53 \\
\hline 60 & 497.59 & 97.88 & 125.65 & 51.62 & 100.17 & 85.28 & 3 & 13.63 \\
\hline \multicolumn{9}{|c|}{ Max-reduced-risk arc selection rule } \\
\hline 20 & 522.17 & 94.97 & 102.18 & 55.23 & 110.13 & 94.35 & 1.5 & 2.73 \\
\hline 30 & 498.24 & 99.58 & 110.93 & 50.04 & 105.03 & 96.23 & 1.4 & 4.20 \\
\hline 40 & 580.23 & 97.61 & 127.32 & 48.02 & 102.13 & 87.39 & 2 & 6.39 \\
\hline 50 & 651.32 & 98.40 & 129.08 & 49.07 & 102.54 & 87.80 & 2.3 & 9.60 \\
\hline 60 & 499.23 & 98.20 & 126.05 & 51.95 & 100.87 & 85.82 & 3 & 13.66 \\
\hline
\end{tabular}

provides a measure of how much risk reduction is possible, by designing a hazmat transport network and restricting all hazmat shipments to this network. Clearly, under all settings there is a substantial reduction in the risk level. The reduction is highest under the aggregate-risk measure where $R_{1}$ is 17 times higher than $R_{4}$. This is because a network designed with a minimum risk objective would avoid the edges near major places of assembly, whereas carriers may use such edges in the absence of restrictions. As one would expect, costs increase with regulation. The cost level increases significantly under the bilevel model, as compared to the unregulated model, as given by $\mathrm{C}_{1} / \mathrm{C}_{4}$. $\mathrm{C}_{4}$ can be as much as twice $C_{1} . R_{2} / R_{4}$ shows how close the risk level found by the bilevel heuristic is to the lowest risk solution. The network calculated by the heuristic is at most $2.5 \%$ from the lowest risk network. It is, on average, $1.62 \%$ under the max-risk rule and $1.28 \%$ under the max-reduced-risk rule. Thus, a bilevel design can come very close, in terms of risk level, to the (unrealistic) benchmark solution that requires a regulation of all routes. In addition, the bilevel design improves the cost in half of the tests, as shown by $\mathrm{C}_{2} / \mathrm{C}_{4} \cdot \mathrm{R}_{3} / \mathrm{R}_{4}$ gives the improvement of the bilevel decision model over the two-step model. The bilevel model leads to a lower risk level in all the tests. The difference is, on average, $4.47 \%$ and can be as much as $9.98 \%$. On the other hand, $\mathrm{C}_{3} / \mathrm{C}_{4}$ shows that the increase in cost associated with the reduction in risk is not significant. $\mathrm{C}_{4}$ is on average higher than $\mathrm{C}_{3}$ by $6.3 \%$ under the max-risk rule and $1.85 \%$ under the max-reduced-risk rule. In general, the max-reduced-risk rule leads to better designs than the max-risk rule.

\subsubsection{Random test problems}

In an effort to test the algorithm on more problem instances, we use the Ravenna network as a testbed to generate additional instances by generating OD pairs randomly from the 105 vertices of the network. We set the number of OD pairs $K=20,30,40,50,60$. For each value of $K$, we fix the number of distinct origins and the number of times each origin repeats, as given in Table 2. For example, the first row of Table 2 suggests that when $K=20$, there are five distinct origin vertices. The first vertex is the origin of five commodities, the second is the origin of six commodities, etc. Then, we generate the origins and destinations randomly. For each instance, let $S$ be the set of origins generated so far; the next origin $i$ is generated randomly in $V-S$, and the destination is generated randomly in $V-\{i\}$. The demand for each OD pair is generated uniformly in $[10,100]$ and rounded to the nearest integer. For each value of $K$, we generate 10 random instances. All instances are generated before testing, and the same instances are used throughout.

Tables 3a,b and 4 give the detailed and summary results on the random instances. All tests are performed using the aggregate-risk measure and both the max-risk and max-reduced-risk arc selection rules.

Both the detailed and the summary results support the observations from the first instance with actual data. All the heuristic network solutions are stable. The reduction in risk due to regulation is, in most cases, more than fourfold $\left(\mathrm{R}_{1} / \mathrm{R}_{4}\right)$. The heuristic finds good quality solutions (low risk), when compared to the over-regulated model. The heuristic finds the lowest possible risk in half of the tests. On average, the risk level is within $2 \%$ of the lowest risk. Moreover, the bilevel design improves the cost level over the over-regulated model in most cases. The reduction in risk over the two-step model is substantial and increases as the number of commodities increases. However, the low risk figures are achieved at the expense of a significant increase in cost. Table 4 indicates that when one moves from the 
Table 5

35-commodity instance results with biobjective function using the aggregate risk measure and the max-reduced-risk arc selection rule

\begin{tabular}{rrrrrrrrr}
\hline$\alpha$ & $\mathrm{R}_{1} / \mathrm{R}_{4}$ & $\mathrm{R}_{2} / \mathrm{R}_{4}$ & $\mathrm{R}_{3} / \mathrm{R}_{4}$ & $\mathrm{C}_{1} / \mathrm{C}_{4}$ & $\mathrm{C}_{2} / \mathrm{C}_{4}$ & $\mathrm{C}_{3} / \mathrm{C}_{4}$ & Iter & $\mathrm{CPU}$-time \\
\hline 0.00 & 1729.38 & 98.20 & 103.11 & 56.62 & 130.00 & 98.56 & 2 & 5.47 \\
0.01 & 1678.11 & 96.97 & 100.00 & 57.51 & 101.30 & 100.00 & 3 & 7.22 \\
0.05 & 1678.11 & 97.00 & 100.00 & 57.51 & 101.28 & 100.00 & 4 & 8.72 \\
0.10 & 1510.13 & 119.27 & 255.76 & 59.97 & 91.93 & 81.03 & 2 & 5.76 \\
0.25 & 581.37 & 55.27 & 195.39 & 78.49 & 112.06 & 91.58 & 7 & 13.87 \\
0.50 & 520.99 & 99.91 & 137.59 & 85.11 & 99.99 & 95.34 & 3 & 7.58 \\
\hline
\end{tabular}

Table 6

Summary results on the random test problems with biobjective function, using the aggregate risk measure and the max-reduced-risk arc selection rule

\begin{tabular}{|c|c|c|c|c|c|c|c|c|c|}
\hline$\alpha$ & $K$ & $\mathrm{R}_{1} / \mathrm{R}_{4}$ & $\mathrm{R}_{2} / \mathrm{R}_{4}$ & $\mathrm{R}_{3} / \mathrm{R}_{4}$ & $\mathrm{C}_{1} / \mathrm{C}_{4}$ & $\mathrm{C}_{2} / \mathrm{C}_{4}$ & $\mathrm{C}_{3} / \mathrm{C}_{4}$ & Iter & CPU-time \\
\hline \multirow[t]{5}{*}{0.01} & 20 & 564.56 & 97.9 & 108.61 & 57.37 & 95.15 & 89.8 & 2.7 & 4.10 \\
\hline & 30 & 479.93 & 99.12 & 110.5 & 55.63 & 97.4 & 94.79 & 3.1 & 6.71 \\
\hline & 40 & 568.63 & 97.58 & 123.85 & 50.69 & 93.25 & 88.45 & 3.3 & 9.70 \\
\hline & 50 & 623.58 & 97.95 & 128.94 & 56.25 & 97.81 & 92.63 & 3.6 & 13.04 \\
\hline & 60 & 521.06 & 96.85 & 132.86 & 57.72 & 95.89 & 90.54 & 4.7 & 19.24 \\
\hline \multirow[t]{5}{*}{0.05} & 20 & 548.56 & 96.56 & 161.96 & 62.2 & 100.42 & 91.85 & 2.9 & 4.33 \\
\hline & 30 & 471.35 & 99.22 & 140.54 & 58.44 & 97.74 & 93 & 3.4 & 7.10 \\
\hline & 40 & 551.25 & 95.96 & 136.35 & 55.5 & 96.06 & 88.91 & 3.8 & 10.67 \\
\hline & 50 & 609.46 & 97.35 & 137.66 & 58.89 & 99.59 & 94.16 & 4.2 & 14.53 \\
\hline & 60 & 514.46 & 96.75 & 127.97 & 61.83 & 100.35 & 95.84 & 6.3 & 23.54 \\
\hline \multirow[t]{5}{*}{0.10} & 20 & 501.19 & 95.39 & 159.77 & 69.35 & 103.57 & 93.92 & 1.9 & 3.30 \\
\hline & 30 & 425.77 & 96.61 & 160.92 & 62.45 & 93.16 & 84.03 & 3.8 & 7.79 \\
\hline & 40 & 527.9 & 99.64 & 179.39 & 58.65 & 94.82 & 83.73 & 2.5 & 8.23 \\
\hline & 50 & 568.36 & 100.44 & 175.66 & 61.4 & 95.38 & 84.71 & 3.2 & 12.33 \\
\hline & 60 & 488.29 & 96.64 & 152.63 & 64.92 & 97.31 & 89.63 & 4.3 & 18.03 \\
\hline \multirow[t]{5}{*}{0.25} & 20 & 436.65 & 95.41 & 149.04 & 76.99 & 101.29 & 95.49 & 3.9 & 5.22 \\
\hline & 30 & 357.79 & 98.16 & 159.95 & 69.39 & 91.23 & 82.99 & 5.3 & 9.84 \\
\hline & 40 & 368.67 & 87.71 & 169.49 & 71.76 & 99.7 & 83.77 & 8.2 & 18.48 \\
\hline & 50 & 445.98 & 102.59 & 204.37 & 70.83 & 93.46 & 81.84 & 6.7 & 20.20 \\
\hline & 60 & 360.56 & 85.09 & 166.15 & 75.56 & 100.78 & 87.13 & 9.8 & 33.02 \\
\hline \multirow[t]{5}{*}{0.50} & 20 & 287.37 & 94.69 & 124.84 & 86.11 & 100.94 & 96.88 & 6.5 & 7.87 \\
\hline & 30 & 230.33 & 87.73 & 131.25 & 86.34 & 101.69 & 93.43 & 9.3 & 15.68 \\
\hline & 40 & 198.71 & 72.76 & 110.5 & 89.63 & 106.34 & 97.64 & 11.4 & 25.15 \\
\hline & 50 & 294.32 & 91.36 & 157.73 & 84.22 & 99.92 & 91.71 & 10.7 & 29.73 \\
\hline & 60 & 257.92 & 78.35 & 129.82 & 87.12 & 103.75 & 95.16 & 11.6 & 38.12 \\
\hline
\end{tabular}

unregulated case to the bilevel case, the five-fold reduction in risk comes with a two-fold increase in cost. Whether this cost increase is acceptable or not depends on the decision makers. In the next section, we introduce a biobjective model that would allow a decision maker to apply explicit cost/risk trade-offs to the design problem.

\subsection{Phase 2: a biobjective-bilevel model}

Although the main concern of the government may be public risk, it is reasonable to assume that they would be somewhat concerned about the financial cost imposed on the industry. In this section, we extend the bilevel model to account for the cost/risk trade-off by including transport costs in the first-level objective. The biobjective-bilevel model allows decision makers to trade off risk and cost. 

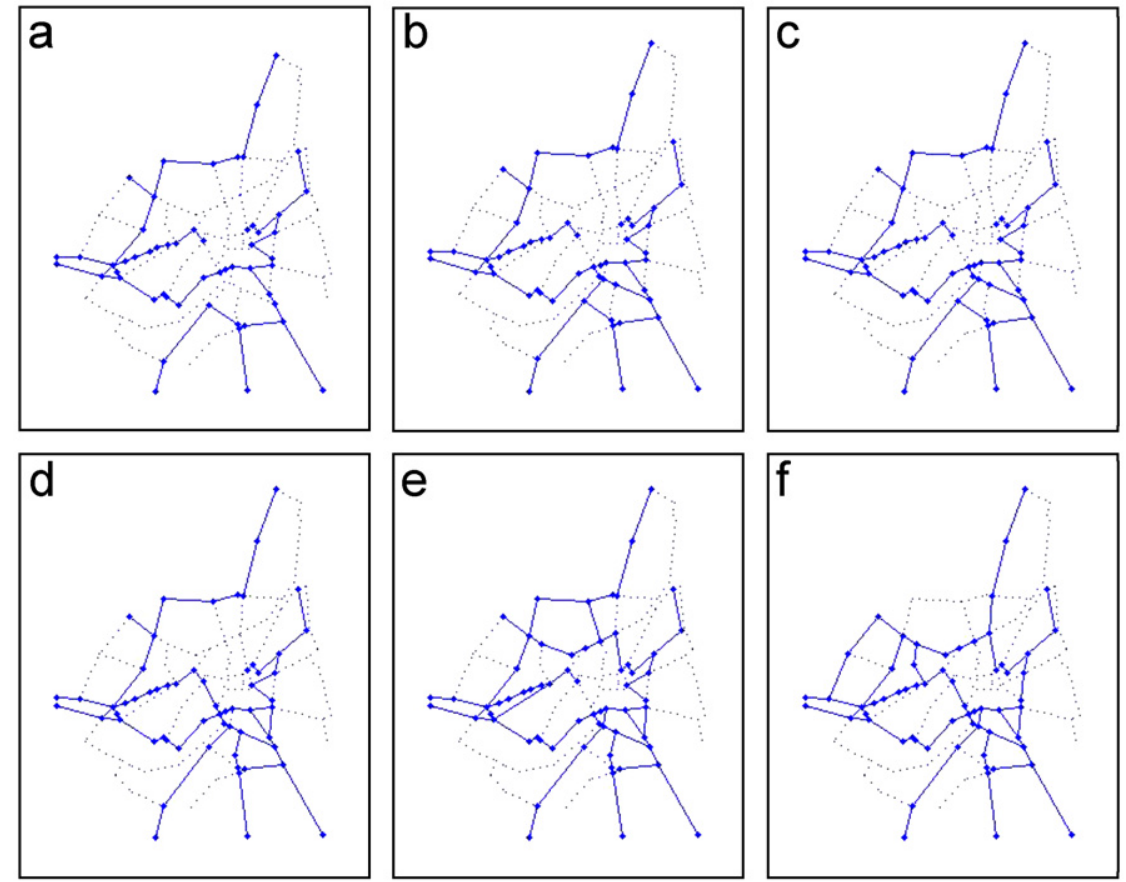

Fig. 3. Network solution of the 35-pairs example.

Let $\alpha \in[0,1]$ be a parameter that indicates the relative importance of the risk and cost objectives for the government. The outer objective of the bilevel model becomes

$$
\min \sum_{k \in\{1 \ldots K\}} \sum_{(i, j) \in A}\left(d_{k} r_{i j k}+\alpha d_{k} c_{i j k}\right) x_{i j k}
$$

The heuristic is modified by changing the objective of ODP to (7). In what follows, we refer to the weighted sum of risk and cost as the weighted risk. The heuristic finds a network solution on which the weighted risk calculated by solving ODP is equal to the actual weighted risk resulting from the solution of IDP. To check the stability of the heuristic network, NF_check is solved with a weighted objective function. We perform tests for $\alpha=0.01,0.05,0.10,0.25,0.50$.

Table 5 gives the results for the actual instance, and Table 6 gives the summary results for the random instances generated on the Ravenna network. Each row gives the results for some value of $\alpha$. Fig. 3 shows the solutions of the actual instance under each value of $\alpha$ including value 0 . In the tables, $R_{2}$ and $R_{4}$ correspond to the risk portion of the weighted risk in the over-regulated and bilevel scenarios. Consequently, $\mathrm{R}_{2}$ is no longer a valid lower bound to $\mathrm{R}_{4}$. All tests are performed using the aggregate-risk measure and the max-reduced-risk arc selection rule.

As $\alpha$ increases, one can make the following observations based on the experiment:

- the reduction in risk over the unregulated case becomes less significant but still substantial,

- risk levels move farther away from the over-regulated scenario,

- the reduction over the two-step model becomes more significant,

- the network becomes denser,

- the cost improves and comes closer to the cost of the unregulated model and even closer to the cost of the two-step model.

It seems that the biobjective-bilevel model can effectively produce a number of network designs and allow the decision makers to trade off risk and cost directly in their final design selection. 


\section{Conclusion}

In this paper, we developed and tested a simple heuristic for a bilevel network design problem for hazmat transportation. The risk-minimizing model explicitly accounts for the cost-minimizing routing decisions that will be made by individual carriers on the given network. The heuristic always generates a stable network solution and protects the government from the worst case scenario when the problem is ill-posed. In this case, the linearization of the bilevel model fails to find a stable solution. The results are encouraging. The heuristic is very fast on a problem instance of realistic size. The risk generated by the shipments on the heuristically designed network is very close to the lowest risk possible, which leads us to conclude that the heuristic solution is close to the optimal bilevel network. Furthermore, the empirical results indicate that a substantial reduction in risk over the unregulated case can be achieved by using the bilevel model to design a hazmat network. As one would expect, this risk reduction comes with an accompanying increase in cost.

We generalized the risk-minimizing bilevel model to a biobjective model by including transport cost in the first level. Clearly the biobjective-bilevel model is a rich decision-support tool that allows for the generation of many good solutions to the design problem. (Given our heuristic for the bilevel problem, we cannot claim that the solutions are Pareto-optimal.) The selection of the final design among these good solutions would depend on the cost/risk trade-offs and the preferences of the decision makers. However, it is easier for a decision maker to compare two designs on their estimated total cost and risk and to select one, than to explicitly articulate a cost/risk utility function. Hence, we suggest varying alpha to solve a number of design problems and then using an interactive procedure that computes the marginal design.

\section{Acknowledgments}

This research was partly conducted at the University of Alberta. We acknowledge the financial support of the Natural Sciences and Engineering Research Council of Canada (NSERC Grant number 25481).

\section{References}

[1] Ben-Ayed O, Blair CE, Boyce DE, LeBlanc LJ. Construction of a real-world bilevel programming model of the highway network design problem. Annals of Operations Research 1992;34(1-4):219-54.

[2] Patriksson M, Rockafellar RT. A mathematical model and descent algorithm for bilevel traffic management. Transportation Science 2002;36(3):271-91.

[3] Gao Z, Wu J, Sun H. Solution algorithm for the bilevel discrete network design problem. Transportation Research Part B 2005;39: 479-95.

[4] Chiou S-W. Bilevel programming for the continuous transport network design problem. Transportation Research Part B 2005;39:361-83.

[5] Yang H, Bell MGH. Models and algorithms for road network design: a review and some developments. Transport Reviews 1998;18:257-78.

[6] Yang H, Bell MGH. Special issue: transport bilevel programming problems: recent methodological advances. Transportation Research B 2001;35:1-4.

[7] Erkut E, Tjandra S, Verter V. Hazardous materials transportation, In: Barnhart C, Laporte G, editors. Operations research handbook on transportation. Amsterdam: Elsevier, forthcoming.

[8] Erkut E, Alp O. Designing a road network for dangerous goods shipments. Computers and Operations Research, forthcoming.

[9] Kara BY, Verter V. Designing a road network for hazardous materials transportation. Transportation Science 2004;38:188-96.

[10] Bard JF. Practical bilevel optimization: algorithms and applications. Dordrecht: Kluwer Academic Publishers; 1999.

[11] Dempe S. Foundations of bilevel programming. Dordrecht: Kluwer Academic Publishers; 2002. 